\title{
A theoretical model of multielectrode DBR lasers
}

\author{
Pan, Xing; Olesen, Henning; Tromborg, Bjarne
}

\section{Published in:}

I E E E Journal of Quantum Electronics

Link to article, DOI:

$10.1109 / 3.14372$

Publication date:

1988

Document Version

Publisher's PDF, also known as Version of record

Link back to DTU Orbit

Citation (APA):

Pan, X., Olesen, H., \& Tromborg, B. (1988). A theoretical model of multielectrode DBR lasers. I E E E Journal of Quantum Electronics, 24(12), 2423-2432. https://doi.org/10.1109/3.14372

\section{General rights}

Copyright and moral rights for the publications made accessible in the public portal are retained by the authors and/or other copyright owners and it is a condition of accessing publications that users recognise and abide by the legal requirements associated with these rights.

- Users may download and print one copy of any publication from the public portal for the purpose of private study or research.

- You may not further distribute the material or use it for any profit-making activity or commercial gain

- You may freely distribute the URL identifying the publication in the public portal

If you believe that this document breaches copyright please contact us providing details, and we will remove access to the work immediately and investigate your claim. 


\title{
A Theoretical Model of Multielectrode DBR Lasers
}

\author{
XING PAN, HENNING OLESEN, AND BJARNE TROMBORG
}

\begin{abstract}
A theoretical model for two- and three-section tunable DBR lasers is presented. The static tuning properties are studied in terms of threshold current, linewidth, oscillation frequency, and output power. Regions of continuous tuning are presented for three-section DBR lasers. We discuss different routes for continuous tuning, when the injection currents to the passive sections are varied, and the limitations on the continuous tuning range are clarified. By proper control of these currents a tuning range of approximately $400 \mathrm{GHz}$ is pre. dicted at $1.55 \mu \mathrm{m}$ wavelength, which is in good agreement with the experimental results published thus far.
\end{abstract}

\section{INTRODUCTION}

$F_{s}$ REQUENCY tunable narrow linewidth lasers are essential components in coherent communication systems. Recently, several laboratories have succeeded in making multielectrode frequency tunable lasers [1]-[6]. Although the spectral linewidth achieved with such devices is rather large compared to discrete external cavity lasers, they do have several advantages, e.g., compact size, high FM efficiency, and low sensitivity to environmental disturbances. In such devices, single-mode operation is realized by a built-in Bragg corrugation in one or more sections. Depending on whether the corrugation is in the active or passive section(s), the laser is either a DFB (distributed feedback) or DBR (distributed Bragg reflector) type.

We have previously reported a theoretical study of the phase-tunable DFB laser [7], which consists of a DFB section and a monolithically integrated passive waveguide section. The principle of frequency tuning in this device is to adjust the phase of the reflected light from the waveguide by injecting carriers into the waveguide section, which in turn changes the refractive index through the free-carrier plasma effect. The frequency tuning is limited to a certain range around the Bragg frequency, which is clamped when the DFB section is operated above threshold. Another disadvantage of this two-section device is that two electrodes do not allow a sufficient control of the oscillation frequency, side mode suppression, and output power. For this purpose three degrees of freedom are re quired, and it is therefore relevant to study three-section devices.

A qualitative analysis of tunable multielectrode lasers

Manuscript received March 31, 1988. This work was supported by the EEC under Contract ST2J-0171-2-DK.

$X$. Pan is with the Electromagnetics Institute, Technical University of Denmark, DK-2800 Lyngby, Denmark.

H. Olesen and B. Tromborg are with the Telecommunication Research Laboratory, DK-2970 Hørsholm, Denmark.

IEEE Log Number 8823596. has been reported by Coldren and Corzine [8]. They point out that a multielectrode DBR laser is superior to a DFB laser for obtaining a large tuning range, because the Bragg frequency is not clamped, as it is in the case of a DFB laser. By current injection to the Bragg section the refractive index and the effective corrugation period can be changed, which in turn changes the Bragg frequency. They also emphasize that a three-section DBR laser with three separate electrodes can provide the desired control of the lasing characteristics.

In this paper, we present a theoretical study of a threesection DBR laser [3], [4], which consists of an active Fabry-Perot section, a passive phase control (PC) waveguide section, and a passive DBR section. The two-section DBR laser [2] is treated as a special case of the threesection device. Our numerical results are in good agreement with the predictions of [8]. Nontunable DBR lasers (i.e., lasers with no electrode on the DBR section) have been studied by Patzak et al. [9], [10] and several other authors, and we have applied a similar approach for calculating the threshold gain, frequency, and linewidth of the DBR modes. The paper is organized as follows. In Section II we give the theoretical background for analyzing the three-section DBR laser and a general discussion on the properties of DBR lasers. Detailed numerical results for both two- and three-section DBR lasers are presented in Section III, and the optimum routes of continuous tuning are discussed. Finally, Section IV gives a summary of the results.

\section{Theoretical BaCKGround}

\section{A. Three-Section DBR Laser Model}

Fig. 1 shows a schematic of a three-section DBR laser. The three sections have separate electrodes and are assumed to be electrically isolated from each other. The PC and DBR sections are made of higher band-gap material than the active section and are therefore passive. When current is injected into one of the passive sections, the carrier density increases and simultaneously the refractive index decreases due to the free-carrier plasma effect. For the DBR section this leads to an increase in the Bragg frequency. However, the absorption also increases due to free carriers and this reduces the amount of feedback to the active section.

The theoretical analysis is based on our transmission line theory for compound cavity semiconductor lasers [11]. For simplicity, we will neglect possible reflections at the interface between the active section and the PC sec- 


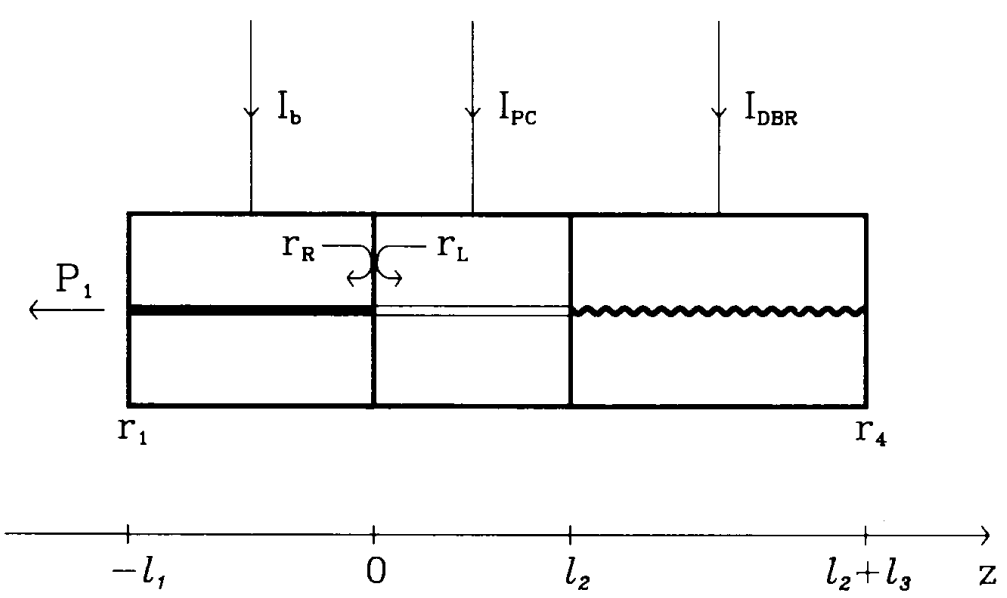

Fig. 1. Three-section tunable DBR laser, $r_{1}$ and $r_{4}$ are the facet reflectivities (amplitude).

tion. A finite reflection coefficient may easily be included if necessary [7]. Setting a reference plane at the interface between the active and the PC section at $z=0$, we write the oscillation condition as

$$
r_{L}\left(\omega, N_{1}\right) r_{R}\left(\omega, N_{2}, N_{3}\right)=1
$$

where

$$
r_{L}\left(\omega, N_{1}\right)=r_{1} \exp \left\{-j 2 k_{1}\left(\omega, N_{1}\right) l_{1}\right\}
$$

is the effective reflectivity of the active section and

$$
r_{R}\left(\omega, N_{2}, N_{3}\right)=r_{\mathrm{DBR}}\left(\omega, N_{3}\right) \exp \left\{-j 2 k_{2}\left(\omega, N_{2}\right) l_{2}\right\}
$$

is the total effective reflectivity of the passive section, both seen from the reference plane. $\omega$ is the angular optical frequency; $N_{1}, N_{2}$, and $N_{3}$ are the carrier densities in the active, the PC, and the DBR sections, respectively; $r_{1}$ is the facet reflectivity at $z=-l_{1}$, and $r_{\mathrm{DBR}}$ is the reflectivity of the DBR section seen towards the right at $z=l_{2}$. The two-section DBR laser (without PC section) just represents the special case with $l_{2}=0$. The PC and DBR sections are assumed to be of the same material composition.

The complex wavenumbers for the active and passive sections are given by

$$
\begin{aligned}
& k_{1}\left(\omega, N_{1}\right)=\frac{\omega}{c} n_{1}\left(\omega, N_{1}\right)+j \frac{1}{2}\left[g\left(\omega, N_{1}\right)-\alpha_{1}\left(N_{1}\right)\right] \\
& k_{i}\left(\omega, N_{i}\right)=\frac{\omega}{c} n_{i}\left(\omega, N_{i}\right)-j \frac{1}{2} \alpha_{i}\left(N_{i}\right) \\
&=\frac{\omega}{c}\left[n_{0}+\Delta n_{i}\left(N_{i}\right)\right]-j \frac{1}{2}\left[\alpha_{0}+\Delta \alpha_{i}\left(N_{i}\right)\right] \\
&(i=2 \text { or } 3)
\end{aligned}
$$

where $c$ is the light velocity in vacuum, $n_{1}$ and $\alpha_{1}$ are the refractive index and internal absorption of the active section, $g\left(\omega, N_{1}\right)$ is the modal gain of the active section, and $n_{0}$ and $\alpha_{0}$ are the refractive index and internal absorption of the passive sections in the absence of carrier injection. The carrier-induced index and absorption changes are expressed as

$$
\begin{aligned}
& \Delta n_{i}\left(N_{i}\right)=\Gamma \frac{d n}{d N} N_{i} \\
& \Delta \alpha_{i}\left(N_{i}\right)=\Gamma \frac{d \alpha}{d N} N_{i}
\end{aligned}
$$

where $\Gamma$ is the confinement factor, and $d n / d N$ and $d \alpha / d N$ are material parameters. The carrier densities relate to the injection currents by

$$
\begin{gathered}
I_{\mathrm{PC}}=e V_{2} R\left(N_{2}\right) \\
I_{\mathrm{DBR}}=e V_{3} R\left(N_{3}\right)
\end{gathered}
$$

where $e$ is the electron charge, $V_{2}$ and $V_{3}$ are the waveguide volumes of the $\mathrm{PC}$ and DBR sections, respectively, and

$$
R(N)=A N+B N^{2}+C N^{3}
$$

is the total spontaneous recombination rate per unit volume.

The expression for $r_{\mathrm{DBR}}\left(\omega, N_{3}\right)$ in (3) is given in Appendix $A$ and is the same as that of a DFB laser [11], [12], except that there is no gain contribution to the imaginary part of the complex wavenumber [cf. (4a)-(4b)]. The Bragg frequency $\omega_{B}$ is taken to be a linear function of $N_{3}$

$$
\omega_{B}=\omega_{B 0}-\Gamma \frac{\omega_{B 0}}{n_{0}} \frac{d n}{d N} N_{3}
$$

where $\omega_{B 0}$ is the Bragg frequency without carrier injection. In all our calculations we have assumed zero reflectivity on the right facet of the DBR section (i.e., $r_{4}=0$, see Fig. 1). A finite facet reflectivity will only have little influence on the tuning properties, since the right traveling wave is attenuated in the DBR section. 


\section{B. Determination of Oscillation Modes and Their Characteristics}

The oscillation condition (1) can be separated into two conditions, one for the gain and one for the phase shift of the field during one round-trip in the total cavity. The gain condition is found from the norm of (1)

$$
\alpha_{\mathrm{th}}\left(\omega, N_{1}\right) l_{1}=\frac{1}{2} \alpha_{2}\left(N_{2}\right) l_{2}-\frac{1}{2} \ln \left|r_{1} r_{\mathrm{DBR}}\left(\omega, N_{3}\right)\right|
$$

where $\alpha_{\mathrm{th}}\left(\omega, N_{1}\right) l_{1}=\operatorname{Im}\left\{k_{1}\left(\omega, N_{1}\right)\right\} l_{1}$ is the net threshold gain for the active section. Equation (11) gives $\alpha_{\mathrm{th}} l_{1}$ as a function of $\omega$ with $N_{2}$ (or $I_{\mathrm{PC}}$ ) and $N_{3}$ (or $I_{\mathrm{DBR}}$ ) as parameters. The modes [i.e., the solutions to (1)] will be located on the curve described by (1) at the positions where the phase condition

$$
2 \frac{\omega n_{1}}{c} l_{1}+2 \frac{\omega n_{2}}{c} l_{2}-\operatorname{Arg}\left\{r_{\mathrm{DBR}}\right\}=2 \pi p, \quad p \text { integer }
$$

is also fulfilled. The expressions for $\alpha_{\mathrm{th}}\left(\omega, N_{1}\right)$ and the refractive index $n_{1}\left(\omega, N_{1}\right)$ in the active section are given in Appendix B. From (11) and (12) we obtain the separate frequency condition

$$
h(\omega)=2 \pi p, \quad p \text { integer }
$$

where

$$
\begin{aligned}
h(\omega)= & 2 \frac{\omega}{c} n_{1}\left(\omega, N_{1}(\omega)\right) l_{1}+2 \frac{\omega}{c} n_{2}\left(\omega, N_{2}\right) l_{2} \\
& -\operatorname{Arg}\left\{r_{\mathrm{DBR}}\left(\omega, N_{3}\right)\right\} .
\end{aligned}
$$

$N_{1}(\omega)$ is the carrier density determined by (11). The $h$ function is useful for analyzing the linewidth and stability properties of the modes [11]. The linewidth of a mode at a given solution to (13) will be inversely proportional to $(d h / d \omega)^{2}$, and a negative value of $d h / d \omega$ indicates that the mode is unstable. The resolution between the frethe mode is unstable. The relation between the frequency condition (13) and the linewidth is an observation [13], [14], which has attracted new attention recently [15], [16].

As shown in [11], the $h$-function can be approximated by

$$
\begin{aligned}
h(\omega)= & h_{0}+\omega \tau_{\text {in }}+2 \frac{\omega n_{2}}{c} l_{2} \\
& +\alpha \ln \left|r_{\mathrm{DBR}}\right|-\operatorname{Arg}\left\{r_{\mathrm{DBR}}\right\}
\end{aligned}
$$

for $\omega$ in the region of the gain peak. Here, $\tau_{\text {in }}$ is the roundtrip time in the active section, $\alpha$ is the linewidth enhancement factor, and $h_{0}$ is a parameter which only depends on the internal state of the active section. [11]

$$
\begin{aligned}
\Delta \nu & =\frac{R|C|^{2}}{4 \pi I_{s}}\left[\operatorname{Re}\left\{\left(1+\frac{\frac{\partial}{\partial \omega} \ln r_{R}}{\frac{\partial}{\partial \omega} \ln r_{L}}\right) C^{*}\right\}\right]^{-2} \\
& =\frac{R|C|^{2}}{4 \pi I_{s}}\left[\operatorname{Re}\left\{C f_{D}^{*}\right\} \frac{d h}{d \omega}\right]^{-2}
\end{aligned}
$$

where $R$ is the spontaneous emission rate into the lasing mode, $I_{s}$ is the photon number, $f_{D}=\left[j(\partial / \partial \omega) \ln r_{L}\right]^{-1}$ is the complex round-trip frequency [11],

$$
C=\frac{1+j \alpha}{1+j \frac{1}{2} G_{\omega}},
$$

$G_{\omega}=v_{g}(\partial g / \partial \omega)$, and $v_{g}$ is the group velocity. Equation (16) agrees with the one used by Patzak et al. for nontunable DBR lasers [9], but is more general. Reference [9] does not take into account the enhancement of spontaneous emission rate due to a nonuniform intensity distribution along the laser cavity or a possible detuning between the Bragg wavelength and the gain peak, but these effects are included in our model.

A graphical illustration of the threshold gain and phase condition for the modes is often used for analysis of nontunable DBR lasers [9], [15]. Fig. 2 shows an example of the threshold gain curve and the $h$-function for the case of a two-section DBR laser. The lengths of the active section $(150 \mu \mathrm{m})$ and the DBR section $(500 \mu \mathrm{m})$ are the same as for the NEC device reported in [2], and the remaining parameter values are listed in Table I. Except for device dimensions no other attempt has been made to fit the parameters to the NEC device. The solid and dashed curves are for $I_{\mathrm{DBR}}=0$ and $100 \mathrm{~mA}$, respectively, and the horizontal axis gives the frequency deviation from the actual Bragg frequency. The modes are marked by dots in Fig. 2(a). The shape of the curves in Fig. 2(a) reflects the frequency dependence of $\left|r_{\mathrm{DBR}}\right|$ and a large pass-band of high reflectivity is observed around the Bragg frequency. As the current to the DBR section is increased, the loss $\alpha_{3}\left(N_{3}\right)$ also increases and we see that the local extrema on each side of the Bragg frequency are "smeared out" and disappear. At the same time the threshold gain near the Bragg frequency becomes higher (i.e., the magnitude of $r_{\mathrm{DBR}}$ decreases). The overall result is a reduced efficiency of the DBR section.

The similarity between the structure of $\alpha_{\mathrm{th}} l_{1}$ [Fig. 2(a)] and the $h$-function [Fig. 2(b)] is due to the dominance of the terms $-(1 / 2) \ln \left|r_{\mathrm{DBR}}\right|$ in (11) and $\alpha \ln \left|r_{\mathrm{DBR}}\right|$ in (15). The influence of the linear term $\omega \tau_{\text {in }}$ in (15) is also clearly seen in Fig. 2(b). The injection of current into the DBR section leads to an upwards shift of the $h$-function. This can be understood in simple terms. The change in the Bragg frequency is determined by the condition $\Delta\left(\omega_{B} n_{3}\right)=0$, i.e.,

$$
\frac{\Delta \omega_{B}}{\omega_{B}}=-\frac{\Delta n_{3}}{n_{3}} .
$$




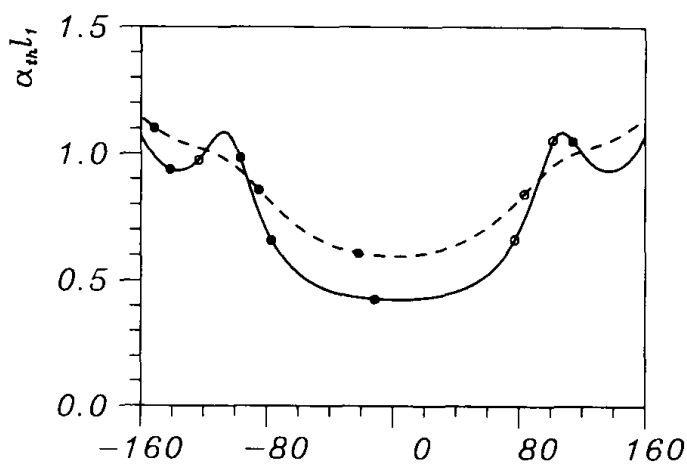

(a)

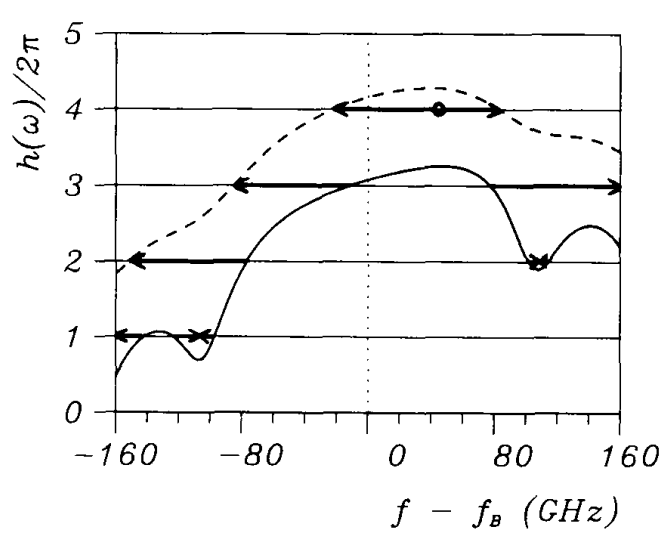

(b)

Fig. 2. (a) Net threshold gain and (b) the $h$-function (14) for a two-section DBR laser. The horizontal axis shows the frequency deviation from the actual Bragg frequency. Solid and dashed curves are for $I_{\mathrm{DBR}}=0$ and $100 \mathrm{~mA}$, respectively. The modes are indicated in (a); closed circles: stable modes, open circles: unstable modes $(d h / d \omega<0)$. The move ments of the modes are indicated by arrows in (b). Parameter values: $l_{1}$ $=150 \mu \mathrm{m}, l_{2}=500 \mu \mathrm{m}, r_{1}=0.565$.

This implies that the relative change in the first term on the RHS of (14) is given by

$$
\frac{\Delta\left(\omega_{B} n_{1}\right)}{\omega_{B} n_{1}}=\frac{\Delta n_{1}}{n_{1}}-\frac{\Delta n_{3}}{n_{3}} .
$$

The refractive index decreases with the carrier density. Since the carrier density is clamped in the active section we have $\left|\Delta n_{1} / n_{1}\right| \ll\left|\Delta n_{3} / n_{3}\right|$. Therefore $\Delta\left(\omega_{B} n_{1}\right) /\left(\omega_{B} n_{1}\right)$ increases and hence $h(\omega)$ is shifted upwards.

In Fig. 2(b) the movement of the modes is indicated. The modes are located at the points where $h(\omega)$ is equal to a multiple of $2 \pi$ and we see that the modes are moving away from the local maxima of $h(\omega)$ as the current is increased. Occasionally, two modes will meet at a local minimum and be annihilated, or two modes may be created at a local maximum and move in opposite directions. This happens when the $h$-function becomes tangential to one of the horizontal lines. The position is known as the phase condition limit [11], [13], and at this point the linewidth of a mode becomes infinite (because the slope of
TABLE I

LIST OF PARAMETER VALUFS

\begin{tabular}{|c|c|c|c|}
\hline Parameter & Symbol & Value & Unit \\
\hline \multicolumn{4}{|l|}{ Active section: } \\
\hline Refractive index & $n_{1}$ & 3.40 & \\
\hline Group refractive index & $n_{g}$ & 3.56 & \\
\hline Internal absorption & $\alpha_{1}$ & 21 & $\mathrm{~cm}^{-1}$ \\
\hline Recombination coefficient & $A$ & $1 \cdot 10^{8}$ & $\sec ^{-1}$ \\
\hline Recombination coefficient & $B$ & $8 \cdot 10^{-17}$ & $\mathrm{~m}^{3} / \mathrm{sec}$ \\
\hline Recombination coefficient & $C$ & $7.5 \cdot 10^{-41}$ & $\mathrm{~m}^{6} / \mathrm{sec}$ \\
\hline Gain curvature & $g_{\omega}$ & $4.85 \cdot 10^{-24}$ & $m^{-1} \sec ^{2}$ \\
\hline Gain coefficient & $g_{N}$ & $9.65 \cdot 10^{-21}$ & $\mathrm{~m}^{2}$ \\
\hline Carrier density at transparency & $N_{0}$ & $1.37 \cdot 10^{24}$ & $\mathrm{~m}^{-3}$ \\
\hline \multicolumn{4}{|l|}{ Extrapolated wavelength of the } \\
\hline & $\lambda_{p}\left(N_{0}\right)$ & 1.60 & $\mu \mathrm{m}$ \\
\hline $\begin{array}{l}\text { Shift of gain peak with carrier } \\
\text { density }\end{array}$ & $d \omega_{p} / d N$ & $2.12 \cdot 10^{-11}$ & $\mathrm{~m}^{3} \sec ^{-1}$ \\
\hline Facet reflectivity & $r_{1}^{2}$ & $32 \%$ & \\
\hline Linewidth enhancement factor & $\alpha$ & 6.6 & \\
\hline Inversion parameter & $n_{s p}$ & 2.2 & \\
\hline Confinement factor & $\Gamma$ & 0.15 & \\
\hline Width of active layer & $w$ & 1.2 & $\mu \mathbf{m}$ \\
\hline Thickness of active layer & $d$ & 0.18 & $\mu \mathrm{m}$ \\
\hline \multicolumn{4}{|l|}{ Passive sections: } \\
\hline Refractive index & $n_{0}$ & 3.40 & \\
\hline Confinement factor & $\Gamma$ & 0.3 & \\
\hline Width of waveguide layers & & 1.2 & $\mu \mathrm{m}$ \\
\hline Thickness of waveguide layers & & 0.23 & $\mu \mathrm{m}$ \\
\hline Internal absorption & $\alpha_{0}$ & $10-20$ & $\mathrm{~cm}^{-1}$ \\
\hline Recombination coefficient & $C$ & $4 \cdot 10^{-41}$ & $\mathrm{~m}^{6} / \mathrm{sec}$ \\
\hline $\begin{array}{l}\text { Index derivative with respect to } \\
\text { carrier density }\end{array}$ & $d n / d N$ & $-5.97 \cdot 10^{-27}$ & $\mathrm{~m}^{3}$ \\
\hline \multicolumn{4}{|l|}{ Absorption derivative with respect } \\
\hline $\begin{array}{l}\text { to carrier density } \\
\text { Brage wavelength without current }\end{array}$ & $d \alpha / d N$ & $2.56 \cdot 10^{-21}$ & $\mathrm{~m}^{2}$ \\
\hline $\begin{array}{l}\text { Bragg waveiength without current } \\
\text { injection }\left(=2 \pi c / \omega_{B 0}\right)\end{array}$ & $\lambda_{B 0}$ & 1.55 & $\mu \mathrm{m}$ \\
\hline Normalised coupling coefficient & $\kappa l_{3}$ & 2.0 & \\
\hline
\end{tabular}

the $h$-function is zero). The dominant mode is the one with the lowest threshold gain and will in general be located near the Bragg frequency [see Fig. 2(a)]. The lowest linewidth is obtained in a region below the Bragg frequency, where the $h$-function has a large and positive slope. Regions where the $h$-function has a negative slope correspond to unstable states.

The example considered in Fig. 2 may be compared with the case of an AR-coated Fabry-Perot laser with feedback from an external grating (cf. [11, Fig. 2(a) and (b)]). The latter configuration is qualitatively very similar to a three-section DBR laser. Both lasers have an active Fabry-Perot section, a phase control option, and a frequency-selective reflector. For the discrete device phase control is performed by changing the cavity length within the order of a wavelength and coarse frequency tuning is done by tilting the grating. The total cavity length and the achievable linewidth reduction can of course be much larger, but at the expense of a more bulky device with high requirements on thermal and mechanical stability.

\section{Tuning Characteristics}

\section{A. The Two-Section DBR Laser}

In Fig. 3 we present the tuning characteristics for the two-section DBR laser considered in Fig. 2. The four figures show the variation of threshold current, frequency, linewidth, and output power, all as a function of the DBR 


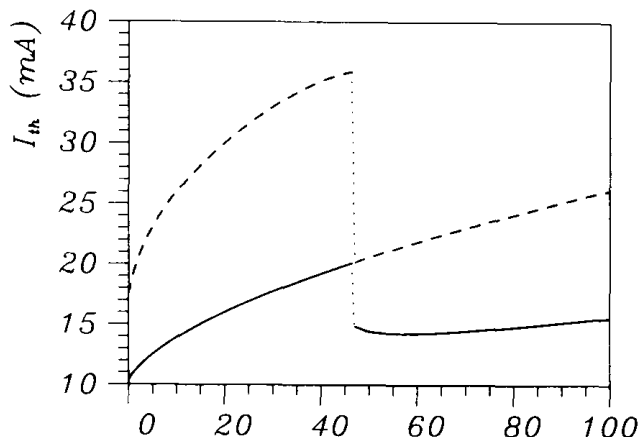

(a)

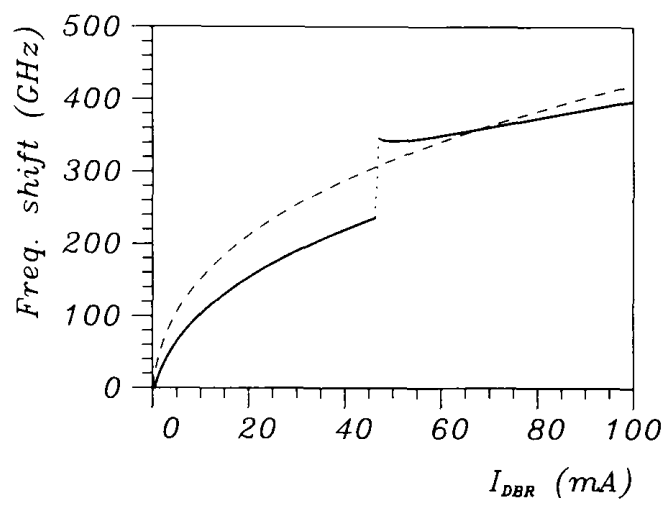

(b)

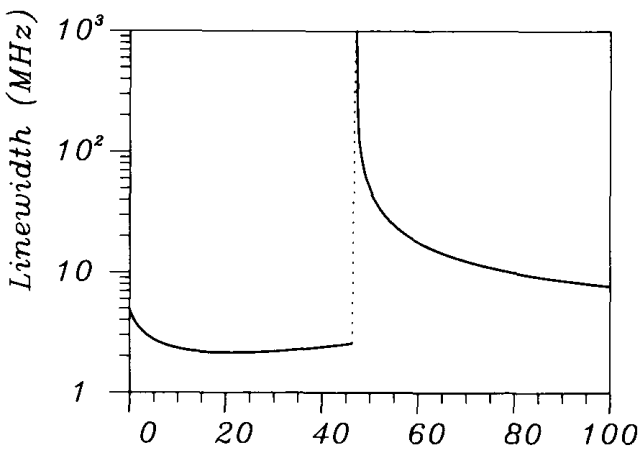

(c)

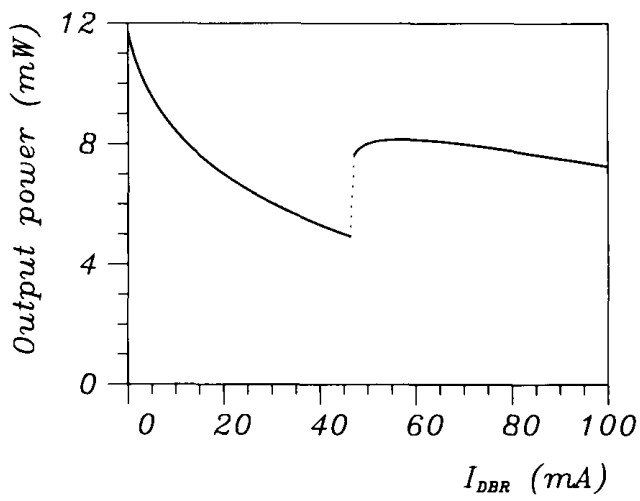

(d)

Fig. 3. Tuning characteristics for a two-section DBR laser (parameters as in Fig. 2) as a function of the DBR current. (a) Threshold currents for the dominant mode (solid curve) and main side mode (dashed curve), (b) frequency of the dominant mode (solid curve) and Bragg frequency (dashed curve), (c) linewidth, and (d) output power.

current. Fig. 3(a) also shows the threshold current of the main side mode and the difference between the two curves can be taken as a measure of the side mode suppression of the device. A new dominant mode is created at $I_{\mathrm{DBR}} \simeq$ $47 \mathrm{~mA}$ and the former dominant mode becomes the main side mode. Before the mode jump occurs, the main mode is moving left on the line $h(\omega) /(2 \pi)=3$ in Fig. 2 (b), and as soon as the new modes are created on the line $h(\omega) /(2 \pi)=4$, the main mode continues from there and moves left. The dashed curve in Fig. 3(b) shows the Bragg frequency. We notice the characteristic sublinear behavior, which is due to the saturation of the carrier density $\mathrm{N}_{3}$ resulting from radiative and Auger recombination. The dominant mode is close to the Bragg frequency but generally has a slower tuning rate [8]. This is clearly seen in Fig. 2(b), where the main mode is always moving left (i.e., towards lower frequency) relative to the Bragg frequency. When the mode gets too far away from the Bragg frequency a mode jump has to occur. This can be eliminated for a three-section device as explained in Section III-B, but is unavoidable for a two-section laser.

The linewidth variation is shown in Fig. 3(c). A minimum linewidth of $2.5 \mathrm{MHz}$ is obtained. Just after the mode jump the linewidth goes to infinity. This happens because the main mode jumps to the phase condition limit [cf. Fig. 2(b)] and the same phenomenon was also observed for the phase-tunable DFB laser [7]. Away from the mode jump region a fairly constant linewidth is observed. The output power is shown in Fig. 3(d). It lies between 6 and $12 \mathrm{~mW}$ with a discrete increase at the mode jump. In general, the power shows a decreasing trend because of the increase in free-carrier absorption in the DBR section. The calculated behavior agrees well with the experimental results of [2].

\section{B. The Three-Section DBR Laser}

In the following we will present similar sets of figures showing the tuning characteristics of a three-section DBR laser. For this analysis we have chosen the cavity lengths reported by Fujitsu in [4], i.e., $l_{1}=300, l_{2}=170$, and $l_{3}=290 \mu \mathrm{m}$. In this case we have the possibility of tuning the two currents $I_{\mathrm{PC}}$ and $I_{\mathrm{DBR}}$ separately, or simultaneously following a given procedure. Initially, we will consider the influence of each of the currents.

Fig. 4 shows the tuning characteristics for variation of the DBR current. Since the total cavity length is larger 

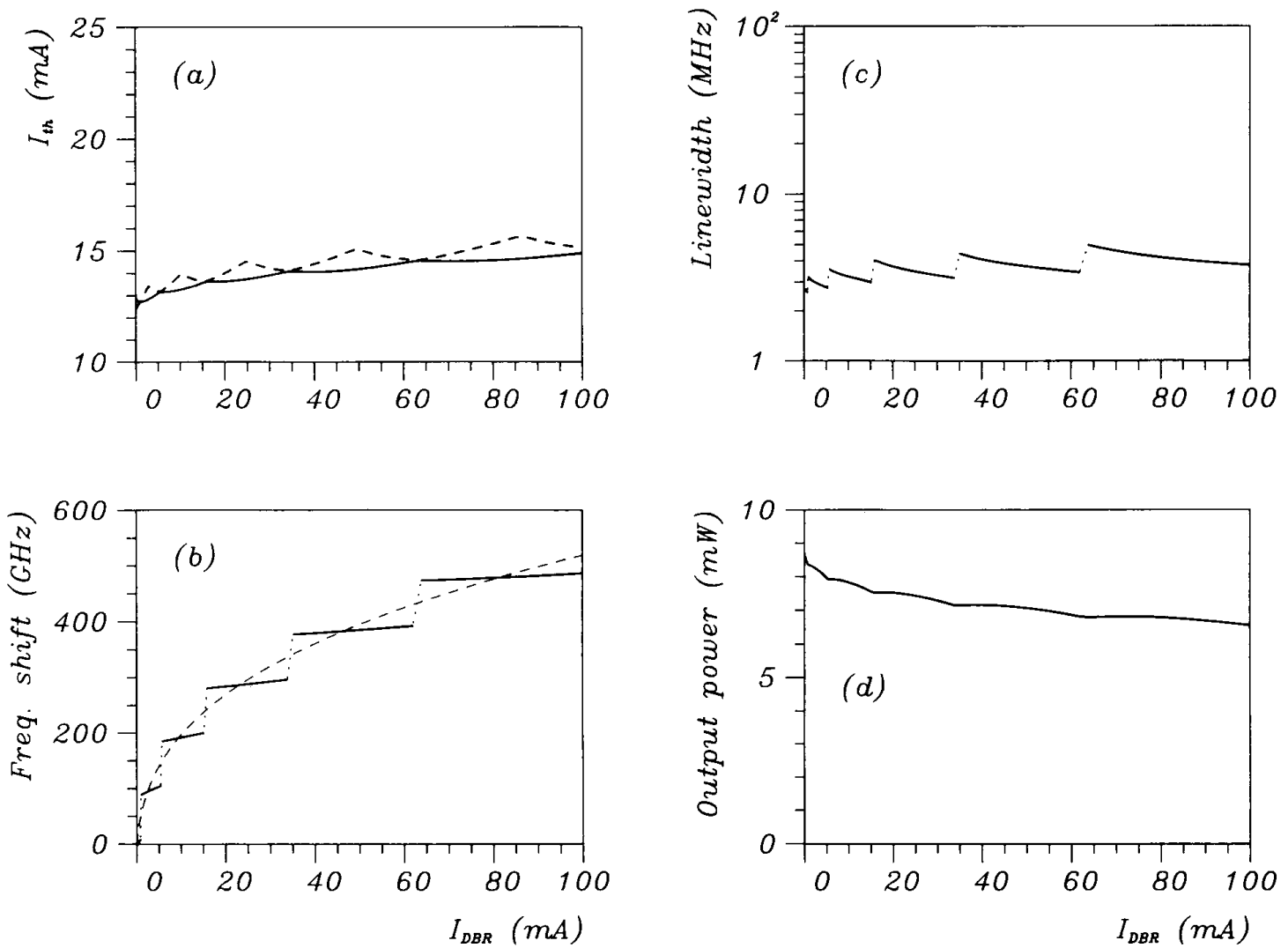

Fig, 4. Tuning characteristics for a three-section DBR laser as a function of the DBR current. Parameter values: $l_{1}=300, l_{2}=170, l_{3}=290 \mu \mathrm{m}$. Legends as in Fig. 3.

than for the two-section device, the linearly increasing part of the $h$-function (not shown) gets a higher slope [ the terms $\omega \tau_{\text {in }}$ and $2 \omega n_{2} l_{2} / c$ in (15)]. The modes are therefore more closely spaced in frequency and more frequent mode jumps are observed. Contrary to the previous example, the mode jumps occur between already existing pairs of modes, so the dominant mode never reaches a phase condition limit. For the same reason, the linewidth variation is rather small. However, our calculation of linewidth is based on a single-mode theory. In practice the laser is likely to oscillate in two (or more) modes close to a mode jump and this will cause a larger linewidth. It is also possible that the the laser will exhibit hysteresis in the region near a mode jump so that the mode can actually be pulled past the limit before a mode jump occurs. Because of the long cavity there are several modes within the main lobe of the Bragg reflector, and the side mode suppression is rather poor. As in the previous example the frequency tunes at a slower rate than the Bragg frequency. The output power varies continuously with a slowly decreasing trend, again because of the increase of free-carrier absorption in the DBR section.

The influence of changing the current to the phase control section is demonstrated in Fig. 5. As the name indicates, the main function of the PC section is to alter the phase of the field which is reflected back to the active section. For a fixed current to the DBR section this enables a tuning across the main lobe of the Bragg reflector as illustrated in Fig. 5(b). The frequency is increasing between the mode jumps and mode jumps become less frequent as $I_{\mathrm{PC}}$ is increased. This is due to saturation of the carrier density $N_{2}$ at high injection level. The frequency tuning is mainly governed by the condition $\Delta\left(\omega n_{2}\right)=0$, cf. (14). Comparing Figs. 4 and 5 we see that the threshold current and output power are more sensitive to $I_{\mathrm{PC}}$ than to $I_{\mathrm{DBR}}$. This agrees with experiments where it is observed that the power drops off faster with $I_{\mathrm{PC}}$ than $I_{\mathrm{DBR}}$ [3].

From the previous discussion it is clear that both of the two currents to the passive sections must be varied in order to obtain continuous tuning over a large frequency range. We have therefore made a calculation of the regions of continuous tuning in the $\left(I_{\mathrm{PC}}, I_{\mathrm{DBR}}\right)$-plane. Because of the increase in Auger recombination and freecarrier absorption at high injection levels, we have restricted both currents to a maximum of $100 \mathrm{~mA}$. The regions are shown in Fig. 6 and are separated by the mode jump boundaries which are indicated as thick solid curves. The regions are obtained as follows. For a given value of $I_{\mathrm{DBR}}$ the slope $d h / d \omega$ at the Bragg frequency is calculated. 

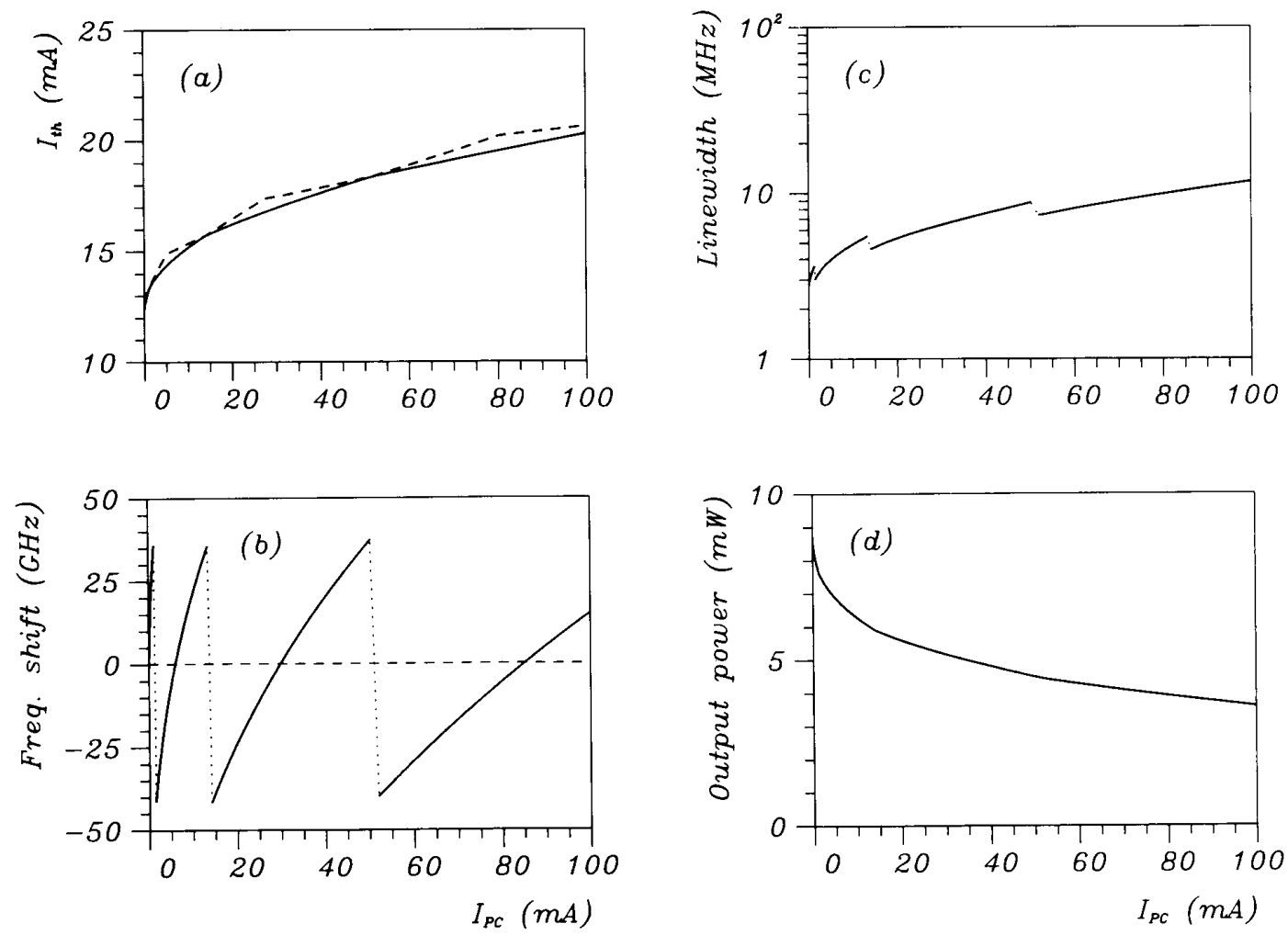

Fig. 5. Tuning characteristics for a three-section DBR laser as a function of the PC current. Same example as in Fig. 4.

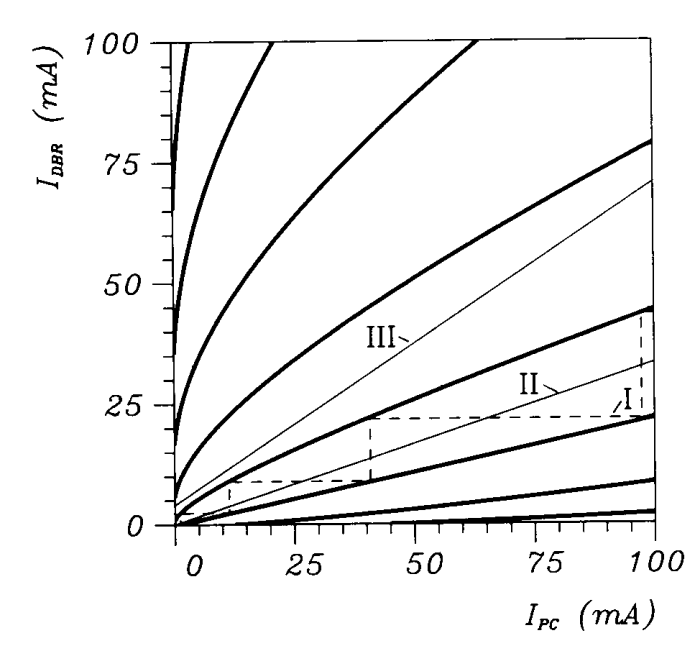

Fig. 6. Regions of continuous tuning for a three-section DBR laser, when the PC and DBR currents are varied. The thick solid curves indicate mode jump boundaries and the thin dashed and solid curves refer to the tuning "routes" I-III, which are discussed in the text.

Because of the long cavity we can assume that the $h$-function varies linearly over a frequency range around the Bragg frequency corresponding to one mode spacing [cf. Fig. 2(b)]. The (approximate) mode jump boundaries are found when two modes are located at $f_{B} \pm \frac{1}{2} \delta f$, where

$$
\delta f=\left[\frac{d h}{d \omega}\right]^{-1}
$$

is the (approximate) mode spacing. Since the threshold gain curve is symmetric around the Bragg frequency [cf. Fig. 2(a)] the two modes will have the same threshold. For constant $I_{\mathrm{DBR}}$ the boundaries are determined by calculating the values of $I_{\mathrm{PC}}$ for which there is a pair of modes at $f_{B} \pm \frac{1}{2} \delta f$. For a shorter device the boundaries may be phase condition limits as shown in Figs. 2 and 3, but for a long cavity with small mode spacing they will always be of the gain condition type. This means that the mode jumps to an already existing mode, which acquires a lower threshold gain than the former one at the boundary. It is of course possible to make a more precise calculation for a shorter cavity, but for the actual case the approximation is sufficiently accurate.

As an interesting result, (16) and (20) imply that the linewidth of the main mode is proportional to the square of the mode spacing. This means that there is a tradeoff between linewidth and side mode suppression, since the side mode suppression will generally become poor if the mode spacing is small (i.e., if the cavity is long). This is a very important consideration for practical design of these devices. 
In the following we will discuss various methods for obtaining a large continuous tuning range. Inspired by the experimental method devised in [4], we tried to calculate the tuning characteristics along the route denoted by $\mathbf{I}$ in Fig. 6. This means increasing one of the currents until just before a mode jump occurs, then increasing the other one, and so on. The frequency tuning is shown in Fig. 7. Here, the horizontal axis shows the current to the PC region, so the vertical curve segments correspond to changing the DBR current, which is not indicated (cf. Fig. 6). Since this is an example of a continuous tuning curve, all other characteristics (threshold current, linewidth, output power etc.) change continuously with current ( $I_{\mathrm{PC}}$ or $\left.I_{\mathrm{DBR}}\right)$, but the qualitative behavior is very similar to the curves of Figs. 4 and 5. Fig. 7 also shows the stepwise increase of the Bragg frequency. It is seen that the actual frequency of the dominant mode changes much less than the Bragg frequency, when $I_{\mathrm{DBR}}$ is varied.

The major disadvantage by following route $I$ is, that we get very close to the mode jump boundaries where the side mode suppression is poor. This can be avoided by following the routes II or III; see Fig. 6 . On these lines $I_{\mathrm{PC}}$ and $I_{\mathrm{DBR}}$ are changed simultaneously, and it is therefore possible to stay clear of the mode jump boundaries over a large range. The relations between the currents are

$$
\begin{aligned}
& I_{\mathrm{PC}}=3 \cdot I_{\mathrm{DBR}} \text {, } \\
& \text { (Route II) } \\
& I_{\mathrm{PC}}=1.5 \cdot I_{\mathrm{DBR}}-6 \mathrm{~mA} . \\
& \text { (Route III) }
\end{aligned}
$$

The resulting tuning characteristics are shown in Fig. 8, here as a function of $I_{\mathrm{DBR}}$. In this particular case there is no advantage in using route III compared to route II, since the tuning range and other characteristics are very similar, and route III requires a higher PC current. However, one might imagine cases where the initial offset of the PC current could be advantageous, for instance if one wants to modulate the PC current with very low injection to the DBR region. In this region the FM efficiency can be very high, with a flat frequency response up to a cutoff frequency determined by the carrier lifetime [17]. In practice, routes II and III will be easier to follow than route I, since they only require the design of a very simple current splitting network.

\section{CONCLUSION}

In this paper we have presented a theoretical model for multielectrode DBR lasers with and without phase control section. The static tuning characteristics have been calculated and methods for maximizing the continuous tuning range have been discussed.

Contrary to a multielectrode DFB laser, where the Bragg corrugation is in the active section, a DBR laser has the possibility of achieving a large frequency tuning range. By current injection to the passive DBR section the refractive index changes due to the free-carrier plasma effect, and this in turn changes the effective corrugation period and the Bragg frequency. Since the carrier density is not clamped (as it is in an active DFB section), the Bragg

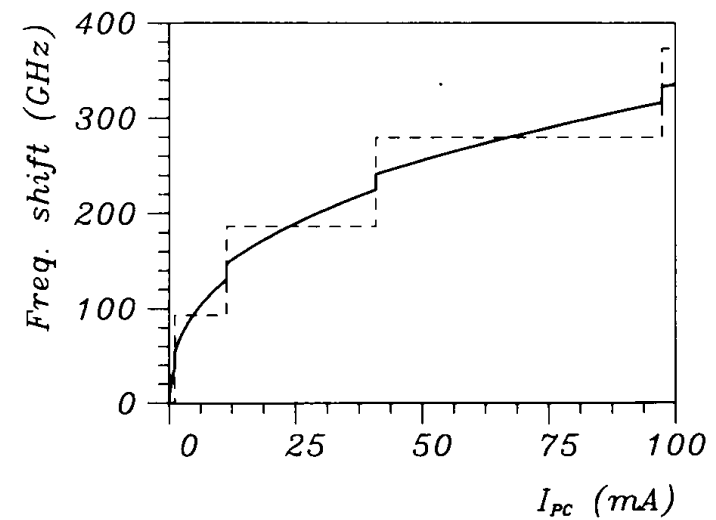

Fig. 7. Frequency tuning characteristics along route $I$ in Fig. 6. Legends as in Fig. 3(b).

frequency can be varied over a large range, typically several hundred $\mathrm{GHz}$. However, Auger recombination and free-carrier absorption strongly limit the efficiency at high injection levels.

The main mode will generally be located close to the Bragg frequency and within the main lobe of the Bragg reflector. Our analysis shows that the tuning rate of the lasing mode will be slower than that of the Bragg frequency, when the DBR current is varied. When the deviation from the Bragg frequency becomes too large, a mode jump to the opposite side of the main lobe will occur, either because a new mode with a lower threshold gain has been created (phase condition jump) or because one of the existing modes acquire a lower threshold gain (gain condition jump). If the total cavity length is large, there will be many modes inside the main lobe, and only gain condition jumps will occur.

In order to avoid mode jumps during tuning of the DBR section, it is necessary to have an additional passive section for phase control. By current injection to this section one can control the phase of the light which is reflected back to the active section, and thereby tune the frequency of the lasing mode around the Bragg frequency. In this work we have calculated the regions of continuous tuning in the $\left(I_{\mathrm{PC}}, I_{\mathrm{DBR}}\right)$-plane, and various routes for continuous tuning have been suggested. Generally, a linear relationship between the two currents gives a large tuning range and the required current splitting is easy to realize in practice. The optimum side mode suppression is obtained when the mode is kept close to the Bragg frequency, but the minimum linewidth is obtained on the low frequency side of the main Bragg lobe. As an important result we have found that the linewidth is proportional to the square of the mode spacing. Thus, a narrow linewidth requires a long cavity with a small mode spacing, whereas a short cavity gives a good side mode suppression (only one or a few modes within the main lobe). This tradeoff must be considered in a practical design.

The typical tuning range by simultaneous control of the PC and DBR currents is on the order of $400 \mathrm{GHz}$, and there is an excellent agreement-both qualitatively and 

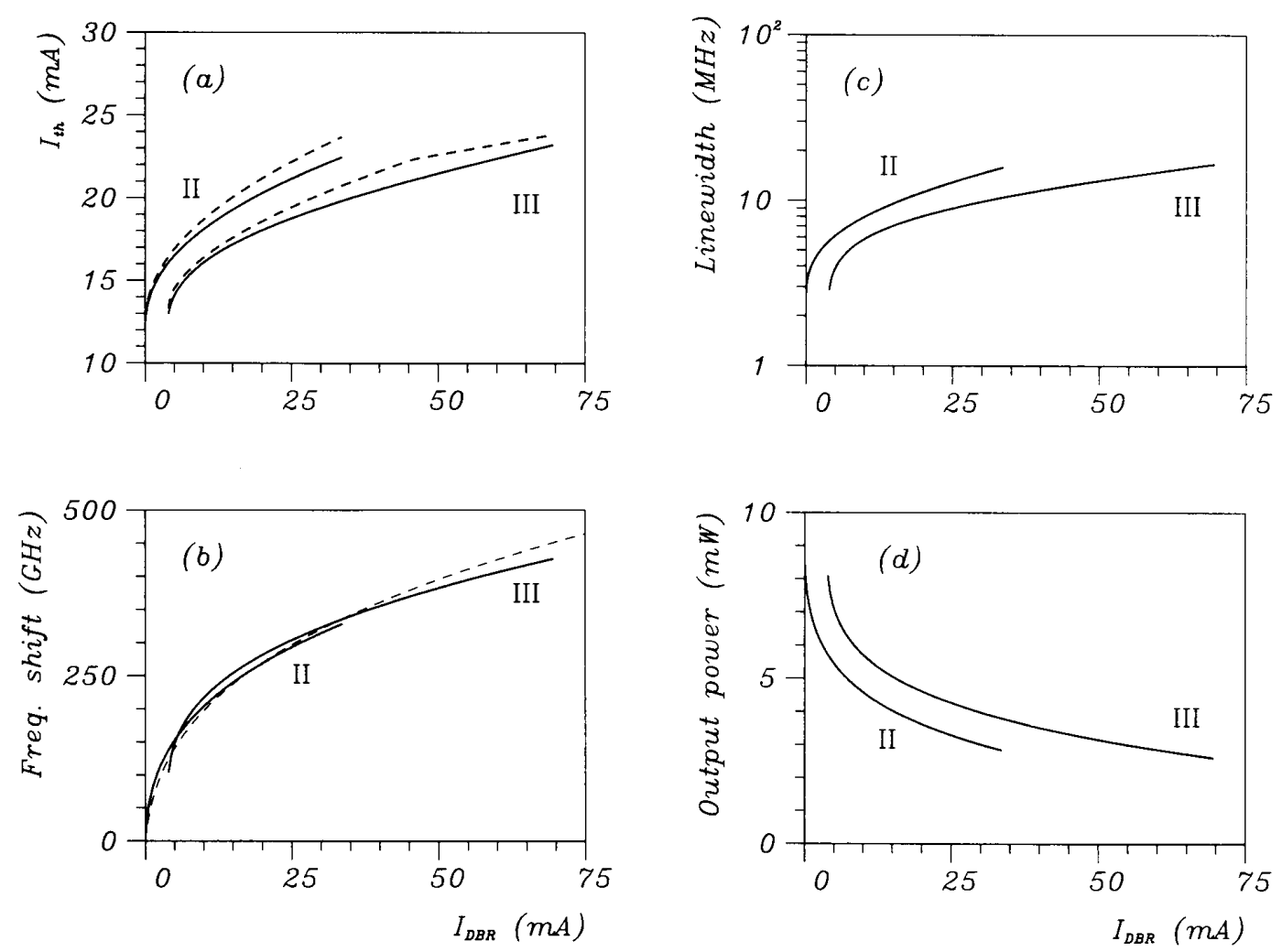

Fig. 8. Tuning characteristics along routes II and III in Fig. 6. Legends as in Fig. 3.

quantitatively - with the experimental results published by NEC and Fujitsu. The present model provides a detailed understanding of the behavior of two- and three-section DBR lasers and our results should be useful for the design of future devices based on these principles.

\section{APPENDIX A}

The expression for the reflectivity of the DBR section is given by [11], [12]

$$
r_{\mathrm{DBR}}\left(\omega, N_{3}\right)=\frac{\frac{\kappa_{c}^{\star}}{\hat{\Gamma}}\left(\rho-\frac{\hat{\Gamma}}{\kappa}\right) e^{\gamma / 3}+\frac{\hat{\Gamma}}{\kappa_{c}}\left(\frac{\kappa}{\hat{\Gamma}}+\rho\right) e^{-\gamma / 3}}{\left(\frac{\hat{\Gamma}}{\kappa}-\rho\right) e^{\gamma / 3}+\left(\frac{\kappa}{\hat{\Gamma}}+\rho\right) e^{-\gamma / 3}}
$$

where

$$
\begin{aligned}
\kappa_{c} & =j \kappa e^{-j \Omega} \\
\rho & =j r_{4} \exp \left\{-j\left(2 \beta_{0} l_{3}+\Omega\right)\right\} \\
\gamma & =\sqrt{\kappa^{2}-\left(k_{3}\left(\omega, N_{3}\right)-\beta_{0}\right)^{2}} \\
\hat{\Gamma} & =-\gamma-j\left(k_{3}\left(\omega, N_{3}\right)-\beta_{0}\right),
\end{aligned}
$$

and $\beta_{0}=\pi / \Lambda$ is the Bragg propagation constant, $\Lambda$ is the period of the (first order) corrugation, $\kappa$ is the coupling coefficient, $r_{4}$ is the facet reflectivity at $z=l_{2}+l_{3}$, and $\Omega$ is the corrugation phase at $z=l_{2}$.

\section{Appendix B}

The relation between the complex wavenumber $k_{1}$ and the frequency and carrier density is given by

$$
\begin{aligned}
\operatorname{Re}\left\{k_{1}\right\}= & \frac{\omega n_{1}\left(\omega, N_{1}\right)}{c} \simeq \frac{\omega_{r} n_{1}\left(\omega_{r}, N_{r}\right)}{c}+\frac{1}{v_{g}}\left(\omega-\omega_{r}\right) \\
& -\frac{1}{2} \alpha \frac{\partial g}{\partial N}\left(N_{1}-N_{r}\right) \\
\operatorname{Im}\left\{k_{1}\right\}= & \alpha_{\mathrm{th}}=\frac{1}{2}\left(g\left(\omega, N_{1}\right)-\alpha_{1}\right) .
\end{aligned}
$$

Here, the product $\omega n_{1}\left(\omega, N_{1}\right)$ is represented by a linear expansion around a reference point $\left(\omega_{r}, N_{r}\right)$ [9], [11], which we have chosen to be $\left(\omega_{B 0}, 2 \cdot 10^{24} \mathrm{~m}^{-3}\right)$. The modal gain is given by

$$
\begin{gathered}
g\left(\omega, N_{1}\right)=g_{N}\left(N_{1}-N_{0}\right)-g_{\omega}\left(\omega-\omega_{p}\left(N_{1}\right)\right)^{2} \\
\omega_{p}\left(N_{1}\right)=\omega_{p}\left(N_{0}\right)+\frac{d \omega_{p}}{d N}\left(N_{1}-N_{0}\right)
\end{gathered}
$$


where $\omega_{p}\left(N_{1}\right)$ is the angular optical frequency of the gain peak, $g_{N}$ is the gain coefficient, $g_{\omega}$ is the gain curvature, and $\omega_{p}\left(N_{0}\right)$ is the (extrapolated) angular frequency of the gain peak for $N_{1}=N_{0}$. The relations (B.1)-(B.4) can easily be inverted, i.e., there is a one-to-one mapping between the $\left(\omega, N_{1}\right)$-plane and the complex $k_{1}$-plane [11].

\section{REFERENCES}

[1] Y. Tohmori, H. Oohashi, T Kato, S. Arai, K. Komori, and Y. Suematsu, "Wavelength stabilisation of $1.5 \mu \mathrm{m}$ GaInAsP/InP bundleintegrated-guide distributed-Bragg-reflector (BIG-DBR) lasers integrated with wavelength tuning region," Electron. Lett., vol. 22, pp. 138-140, Jan. 1986.

[2] S. Murata, I. Mito, and K. Kobayashi, "Spectral characteristics for a $1.5 \mu \mathrm{m}$ DBR laser with frequency-tuning region," IEEE J. Quantum Electron., vol. QE-23, pp. 835-838, June 1987.

[3] —, "Over $720 \mathrm{GHz}(5.8 \mathrm{~nm})$ frequency tuning by a $1.5 \mu \mathrm{m}$ DBR laser with phase and Bragg wavelength control regions," Electron. Lett., vol. 23, pp. 403-405, Apr. 1987.

[4] Y. Kotaki, M. Matsuda, M. Yano, H. Ishikawa, and H. Imai, "1.55 $\mu \mathrm{m}$ wavelength tunable FBH-DBR laser," Electron. Lett., vol. 23, pp. 325-327, Mar. 1987.

[5] Y. Yoshikuni and G. Motosugi, "Multielectrode distibuted feedback laser for pure frequency modulation and chirping suppressed amplitude modulation," J. Lightwave Technol., vol. LT-5, pp. 516-522, Apr. 1987.

[6] T. P. Lee, S. G. Menocal, S. Sakano, V. Valster, and S. Tsuji, "Linewidth and FM characteristics of a distributed feedback laser monolithically integrated with a tunable external cavity," Electron. Lett., vol. 23, pp. 153-154, Feb. 1987.

[7] H. Olesen, X. Pan, and B. Tromborg, "Theoretical analysis of tuning properties for a phase-tunable DFB laser," this issue, pp. 2367-2375.

[8] L. A. Coldren and S. W. Corzine, "Continuously-tunable single-frequency semiconductor lasers," IEEE J. Quantum Electron., vol. QE23 , pp. 903-908, June 1987 .

[9] E. Patzak, P. Meissner, and D. Yevick, “An analysis of the linewidth and spectral behavior of DBR lasers," IEEE J. Quantum Electron., vol. QE-21, pp. 1318-1325, Sept. 1985.

[10] P. Meissner and E. Patzak, "Investigation of linewidth and side-mode suppression for a DBR laser with integrated passive waveguide," Electron. Lett., vol. 22, pp. 340-341, Mar. 1986.

[11] B. Tromborg, H. Olesen, X. Pan, and S. Saito, "Transmission line description of optical feedback and injection locking for Fabry-Perot and DFB lasers," IEEE J. Quantum Electron., vol. QE-23, pp. 18751889 , Nov. 1987.

[12] W. Streifer, R. D. Burnham, and D. R. Scifres, "Effect of external reflectors on longitudinal modes of distributed feedback lasers," IEEE J. Quantum Electron., vol. QE-11, pp. 154-161, Apr. 1975.

[13] B. Tromborg, J. H. Osmundsen, and H. Olesen, "Stability analysis for a semiconductor laser in an external cavity," IEEE J. Quantum Electron., vol. QE-20, pp. 1023-1032, Sept. 1984.

[14] J. Mark, E. Bødtker, and B. Tromborg, "Measurement of Rayleigh backscatter-induced linewidth reduction," Electron. Lett., vol. 21, pp. 1008-1009, Oct. 1985.

[15] R. F. Kazarinov and C. H. Henry, "The relation of line narrowing and chirp reduction resulting from the coupling of a semiconductor laser to a passive resonator," IEEE J. Quantum Electron., vol. QE23, pp. 1401-1409, Sept. 1987.

[16] P. Gallion and G. Debarge, "Relationship between linewidth and chirp reduction in gain-detuned composite-cavity semiconductor lasers," Electron. Lett., vol. 23, pp. 1375-1376, Dec. 1987.

[17] S. Murata, I. Mito, and K. Kobayashi, "Frequency modulation and spectral characteristics for a $1.5 \mu \mathrm{m}$ phase tunable DFB laser," Electron. Lett., vol. 23, pp. 12-14, Jan. 1987.

Xing Pan, for a photograph and biography, see this issue, p. 2375.

Henning Olesen, for a photograph and biography, see this issue, p. 2375.

Bjarne Tromborg, for a photograph and biography, see p. 133 of the February 1988 issue of this JOURNAL. 